\title{
Modified Models Design for Surveillance Radar Electronic Counter-Countermeasure Interaction Training System
}

\author{
Xiaojun Wang ${ }^{1}$, a, Shusheng Yan ${ }^{1}$, Kangying Yin ${ }^{1}$, Xiaoyong $\mathrm{Lu}^{2}$, Peng Zhao ${ }^{1}$ \\ ${ }^{1}$ Simulation Training Center, Air Force Early Warning Academy, Wuhan, Hubei, China. \\ Unit 93897 PLA, Xi'an, Shanxi, China. \\ aguest173@163.com
}

\begin{abstract}
Keywords: Radar, Electronic Counter-Countermeasure, Simulation Modeling, Radar Interaction Training Logic Model.
\end{abstract}

Abstract. On the purpose of radar operators ability improving, for a man-in-the-loop training system a radar interaction training logic (RITL) model is proposed obeying the entity-action-task-interaction (EATI) military concept modeling rules. The radar interactive training space in RITL model consists of entities, activities, rules and interface four components through which the operational ability of radar trainees in electronic counter-countermeasure (ECCM) environment could be characterized. In virtue of the joint use of typical models in radar ECCM system simulation such as radar radiative electromagnetic environment (EME) model, jamming models, radar ECCM models and RITL model, a complete interaction architecture for surveillance radar operators training is illustrated in this paper as well. The preliminary results of experiment show that the operational ability of different trainees could be distinguished which indicates the archetypal system being efficient and acceptable.

\section{Introduction}

To improve the electronic count-countermeasure (ECCM) ability of ground-site surveillance radar operators, models design in radar ECCM interaction training system becomes vital and urgent in recent years. In this field three types of models are reviewed and proposed in reference[1-5]. The first is to characterize radar radiative electromagnetic environment (EME) through pulse description word (PDW) being a radar parameters description set with the elements denoted by radio frequency, pulse width, pulse repetition interval, pulse amplitude, time of arrival, angle of pulse arrival, etc[2,3]. The second is for jamming environment modeling including barrage jamming and decoy jamming models[4,5]. Noise frequency-modulated and amplitude-modulated barrage are two typical suppression jamming, besides, decoy jamming has different types such as velocity decoy, azimuth decoy and range decoy, etc[4]. The third type is radar ECCM interactive models primarily analyzed through frequency and power countermeasure angles[1].

Whereas, the design for these models is not complete to a man-in-the-loop training system since no model is suitable for radar operators training process. In the field of campaign simulation, a method called entity-action-task-interaction (EATI) military concept modeling is widely used[6-8]. In this paper, a radar interaction training logic(RITL) model is proposed obeying the EATI modeling rules, in which a radar interaction training space is defined including four elements such as entities, activities, rules and interface. In virtue of RITL model, the operational ability of radar trainees in ECCM environment could be characterized. This paper is organized by four parts. In section 2 typical models for campaign simulation system in former literatures are reviewed. Then the definition of RITL model and its character is presented plus a modified interaction architecture for radar operators training system is illustrated in section 3 and section 4, respectively. In section 5 the experiment and results are analyzed in which a long-range L-band primary surveillance radar is adopted as the example with a simulated frequency modulated noise barrage jamming environment. Through the simulation for RITL model and radar ECCM model, the operation ability evaluation results of radar trainees is obtained, showing that the modified model interactive structure is efficient and accepted and could be adapted to accomplish an archetypal pattern. 


\section{Typical Models for Rader ECCM system}

Typical models design has three components[1]. The first is radar PDW model[2,3], the second are jamming environment models, and the last are radar ECCM models. These three components have two kinds of logical relations: one is electromagnetic information radiation and reconnaissance, the other is countermeasure and counter-countermeasure.

\section{Radar Electromagnetic Environment Model}

PDW consists of such parameters as Radio Frequency (RF), Pulse Width (PW), Time of Arrival (TOA), Pulse Repetition Interval (PRI), Pulse Amplitude (PA), Angle of Arrival (AOA), i.e., $\mathrm{PDW}=(\mathrm{RF}, \mathrm{PW}, \mathrm{TOA}, \mathrm{PRI}, \mathrm{PA}, \mathrm{AOA})$. In which three typical feature parameters RF, PW and PRI have similar models[3].

Let $\Theta$ represents any parameter among RF, PW and PRI, we have

$$
\Theta=\Theta_{0}+\delta_{\Theta} \times \gamma+\Delta_{\Theta} \times \Pi(-1,1)
$$

Where $\Theta_{0}$ is current operating parameter $\Theta$ of radar, $\delta_{\Theta}$ is the random frequency fluctuation of the radar oscillator, $\gamma$ shows a normal random number with mean value zero and a certain value of variance, $\Delta_{\Theta}$ represents the changing range of parameter $\Theta, \Pi(-1,1)$ is an independent random number obeying uniform distribution within the range $[-1,1]$.

\section{Jamming Environment Modeling}

Being a typical type of jamming, the real part signal model of noise frequency modulation (FM) barrage is given as[4]

$$
u_{\mathrm{fm}}(t)=U_{\mathrm{j}} \cos \left[\omega_{\mathrm{j}} t+2 \pi K_{\mathrm{fm}} \int_{0}^{t} u(\tau) \mathrm{d} \tau+\varphi\right]
$$

Where, $U_{\mathrm{j}}$ is the amplitude of jamming, $\omega_{\mathrm{j}}$ represents the center frequency of FM barrage noise, $K_{\mathrm{fm}}$ is the modulation slope, $\varphi$ shows a statistic variable obeying uniform distribution among $[0,2 \pi]$ and being independent to spot frequency signal $u(t)$. If the purpose of jamming is to deceive the radar target detector and tracking system, then to prevent them from distinguishing true target echoes and tracing target's correct tracks, this kind of jamming is named decoy.

\section{Radar ECCM Models}

This models unit includes power ECCM, frequency ECCM models. According to the primary radar equation and radar operation principle, for the radar-to-jammer power ECCM, the distance transmission loss between radar receiver and jammer in the air just needs to be considered. Under this condition, typical radar equation could be simplified as

$$
P_{r}=P_{j} G_{j} G_{r} \lambda_{r} \lambda_{j} /(4 \pi)^{2} R^{2}
$$

Where $P_{r}$ is the receiving power from the jammer of one radar equipment, $P_{j}$ is the jamming incident power, $G_{j}$ indicates the jammer antenna gain, $G_{r}$ shows the radar receiver antenna gain, $\lambda_{r}$ and $\lambda_{j}$ are wavelength of radar receiver and jammer transmitter respectively. As for frequency ECCM model, the kernel is to determine the mixing field of bandwidth between jammer and radar receiver. The equation is shown as

$$
P_{j r}=P_{r} \bullet R_{\text {mix }}
$$

In which, $P_{r}$ is the received jamming power from Eqn.(3), $R_{\text {mix }}$ is a percentage representing the size of frequency mixing field.

\section{Radar Interaction Training Logic(RITL) Model}

Being an abstraction for radar operation training in ECM environment, RITL model is a normalized mathematical logical description for every variable or parameter, input and output data in radar interaction training space. It has typical features of military concept model such as not directly connected with radar operation training scenario in some area or time period, independent with software programming, comprising a complete set of all possible training scenarios, rational 
for objective operation logistic rules[6], etc.

\section{Definition of RITL Model}

RITL model is a type of military concept model. The radar interaction training space is defined as $S=$ \{entities, activities, rules, interface\}. The training space $S$ has general linguistic and logical features. The establishment of $S$ is based on EATI military concept modeling standard, in which the explanation for four elements of $S$ is shown below.

\section{Elements in RITL Model}

\section{Entities}

Entities denote all possible elements such as radar cabinets, units, plug-in units, switches, push buttons, etc., that maybe used in radar operation training space $\mathrm{S}$.

\section{Activities}

Activities are a subset comprising two elements, one being actions such as switch on, switch off, push-in, pull-out and select, etc., the other being operations such as radar terminal open, radar receiver close, radar adaptive frequency hopping, radar anti-decoy, etc. Moreover, the actions belong to the subsets of operations.

\section{Rules}

Similarly, rules are a subset consisting of three elements: trigger condition of every action or operation, activities order and results of every activity. In which, trigger conditions denote initiate states of all relevant entities connecting with current entity. Activities order represents a logical set combined with elements such as at the beginning, before, after, or, and, finally, etc. Results of activities indicate a linguistic set having such elements as results of actions and results of operations.

\section{Interface}

Interface means the interaction relation between training space $S$ and other models outside, which includes input and output data.

\section{Modified Interaction Architecture}

Under a visual ECM environment, a modified electromagnetic interaction architecture for radar operators training is designed as Fig.1.

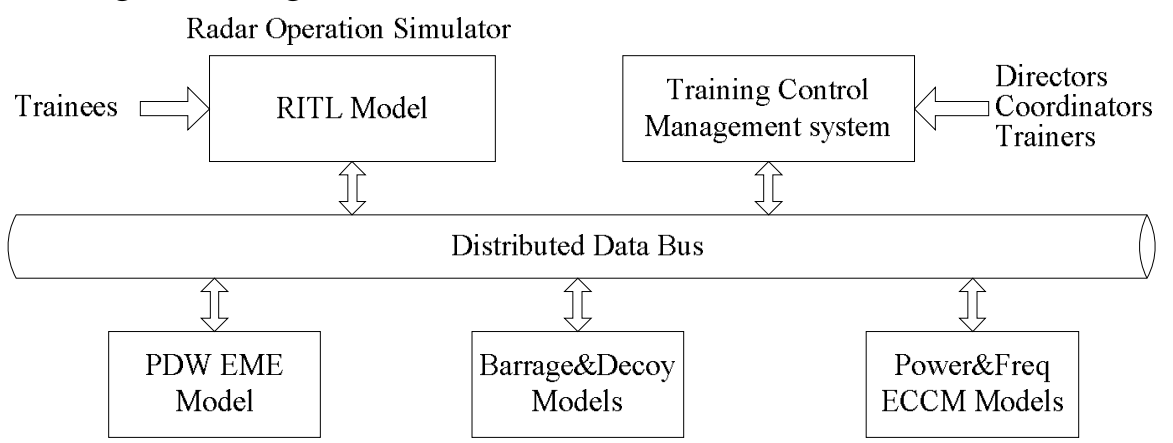

Fig.1. An Modified Interaction Architecture with RITL Model

Compared to original architectures represented by references[1,4], RITL model is added into the new architecture. Therefore, there are similar two level in Fig.1. One is the models level including PDW EME models, jamming models, power ECCM and frequency ECCM models, plus RITL model. The former three models are designed for ECM and ECCM system simulation, while only RITL model serves the man-in-the-loop. Through such a RITL model the radar operators could learn the knowledge and ability to operate simulated radar equipment normally under a complicated ECM scenarios. The other is application level having radar operation simulation, training control and management system. The later comprises: 1) A jamming simulator controlled by directors, coordinators or trainers; 2) An EME analysis, control and management being the headquarters for whole training system, from which the system time calibration and simulation procedures could be controlled by training scenario; 3) A situation displayer. 


\section{Simulation and Results}

In Fig.1, for the interaction architecture we have had the simulation results on three typical models being presented in subsection 4.1[1,3-4]. As for the modified model, the RITL model under barrage jamming environment is taken as an example and the corresponding simulated result is shown in subsection 4.2.

\section{Typical Models Simulation Results}

Radar PDW EME model of Eqn.(1), barrage jamming model in Eqn.(2) and radar ECCM models of Eqn.(3) and Eqn.(4) are included.

\section{Radar PDW EME Model and Jamming Model Results}

The equipment adopted in experiment is a new generation long-range L-band primary surveillance radar designed for en-route control area with complex air traffic situations, which has two types of pulse width $3 \mathrm{us}$ and $300 \mathrm{us}$. Besides, it has two different radio frequency $1270 \mathrm{MHz}$ and 1330MHz. Using Matlab simulation tool, the result of histogram for RF pulses in PDW EME is shown as Fig.2. In Eqn.(1), the results of radar PRI and PW model simulating are similar to Fig.2.

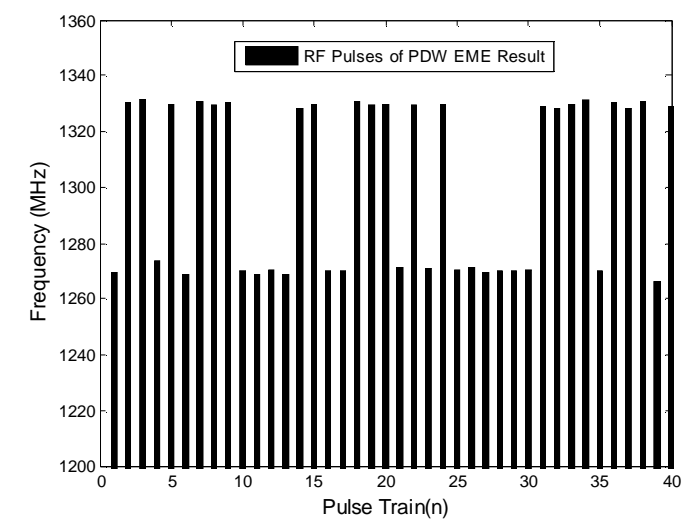

Fig.2. RF Pulses of PDW EME Result

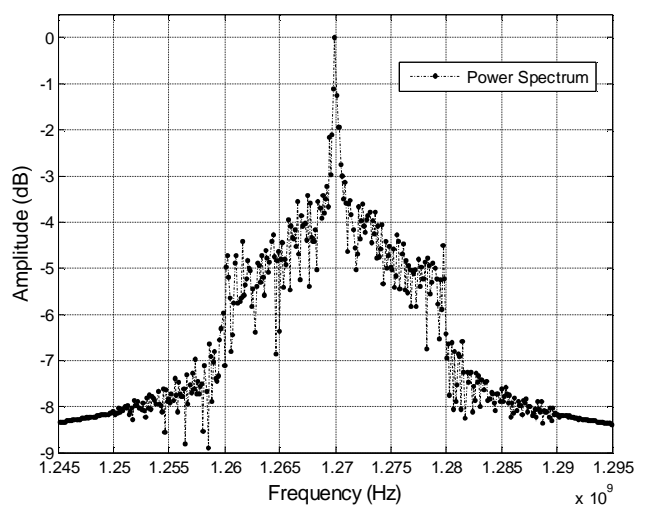

Fig.3. A FM Barrage Result of Jamming $\left(f_{\mathrm{j}}=1270 \mathrm{MHz}\right)$

The power spectrum graph simulated result of jamming environment is shown as Fig.3. Aiming at a model of a radar equipment in $\mathrm{L}$ band within $1200 \mathrm{MHz} 1400 \mathrm{MHz}$, the initiate jamming parameters are designed. For example, the center frequency $f_{\mathrm{j}}=1270 \mathrm{MHz}$, Bandwidth $\Delta f_{\mathrm{n}}=$ $20 \mathrm{MHz}$, the mean power $P_{\mathrm{j}}=1 \mathrm{~W}$. The jamming type parameter is loaded as barrage.

\section{Radar ECCM Simulation Result}

The radar ECCM simulation results comprise radar frequency ECCM and power ECCM.

\section{Result of Radar Frequency ECCM}

The result of radar frequency ECCM is shown as Fig.4, in which three cases are considered. For case one, let jamming working central frequency be $117.5 \mathrm{MHz}, \mathrm{FM}$ bandwidth be $4 \mathrm{MHz}$, radar receiving central frequency be $120 \mathrm{MHz}$ and bandwidth be $5 \mathrm{MHz}$. The jamming energy is attenuated obviously between $113 \mathrm{MHz}$ and $117.5 \mathrm{MHz}$. Beyond $117.5 \mathrm{MHz}$ until $121.5 \mathrm{MHz}$, the jamming energy could efficiently pass through radar receiver described as the dashed line. As for case two, jamming working central frequency is $120 \mathrm{MHz}, \mathrm{FM}$ bandwidth is $4 \mathrm{MHz}$, radar receiving central frequency and bandwidth are $120 \mathrm{MHz}$ and $5 \mathrm{MHz}$, respectively. In Fig.4, the solid line shows the result where primary jamming has received by radar except that being lower than $117.5 \mathrm{MHz}$ or higher than $122.5 \mathrm{MHz}$. In case three, suppose that jamming working central frequency is $120 \mathrm{MHz}, \mathrm{FM}$ bandwidth is $4 \mathrm{MHz}$, radar receiving central frequency is $120 \mathrm{MHz}$ and bandwidth is $9 \mathrm{MHz}$. The corresponding results are shown as the dash-dotted line in Fig.4, where jamming is absorbed by receiver completely. Beyond the jamming bandwidth but within the radar receiver bandwidth, it could be inferred that radar equipment could still normally detect the air targets.

\section{Result of Radar Power ECCM}

In Eqn.(3), let $\lambda_{r}=2.56 \mathrm{~m}, \lambda_{j}=2.5 \mathrm{~m}, G_{j}=G_{r}=20 \mathrm{~dB}, P_{j}$ is normalized as $1 \mathrm{kw}$. In Eqn.(4), supposes that $R_{\text {mix }}$ represents $43.8 \%, 62.5 \%, 100 \%$ in accordance with three cases in Fig.4, 
respectively, the result of jammer residual power simulation is shown as Fig.5. In which, the horizontal axis denotes the distance between jammer and radar site, the vertical axis shows the jamming residual power passing through radar receiver. On the whole, three curves largely obey exponential attenuation function according to different distance.

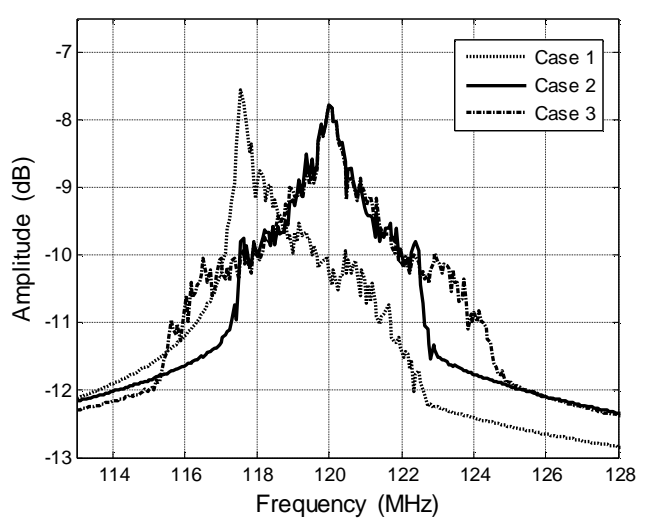

Fig.4. Result of Radar Frequency ECCM

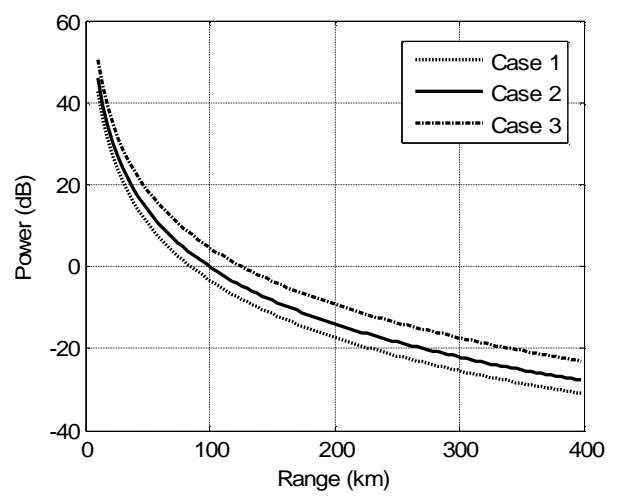

Fig.5. Result of Radar Power ECCM

\section{An Anti-barrage RITL Model Simulation Result}

Given as an example, a RITL model for anti-barrage noise jamming is designed below. In which, the radar is L-band used for long-range primary surveillance and has tens of different radio frequency from $12 \times \times \mathrm{MHz}$ to $13 \times \times \mathrm{MHz}$ with the bandwidth larger than $50 \mathrm{MHz}$.

\section{Definition of Elements in anti-barrage RITL Model}

Entities are denoted as $\mathrm{E}=\{$ monitor cabinet, monitor panel, frequency control buttons, etc. $\}$.

Activities are defined as $\mathrm{A}=\{$ action, operation $\}$, where action $=$ enter, leave, tap, switch on, switch off, push in, push out, etc. $\}$ and operation $=\{$ anti-FM-barrage, anti-AM-barrage, anti-swept-barrage, ...

Rules are shown as $\mathrm{R}=$ \{initiate condition, at first, after, in front of, and, or, finally, etc. $\}$.

Interface is labeled as $\mathrm{I} / \mathrm{O}=$ input, output $\}$, where input $=\{\mathrm{FM}$ barrage image, $\mathrm{AM}$ barrage image, swept barrage image, etc. $\}$ and output $=\{$ ECCM complete, ECCM half complete, ECCM failed\}.

\section{Simulation Result}

On the terminal displayer of L-band radar, the image of FM barrage represented in Fig. 3 is shown as Fig.6. In which, the original point of displayer indicates radar site and within approximately $150 \mathrm{~km}$, there exists numerous ground clutter. Besides, as for azimuth dimension from 30 to 60 degree and within $300 \mathrm{~km}$, there are obvious noise speckle on the screen.

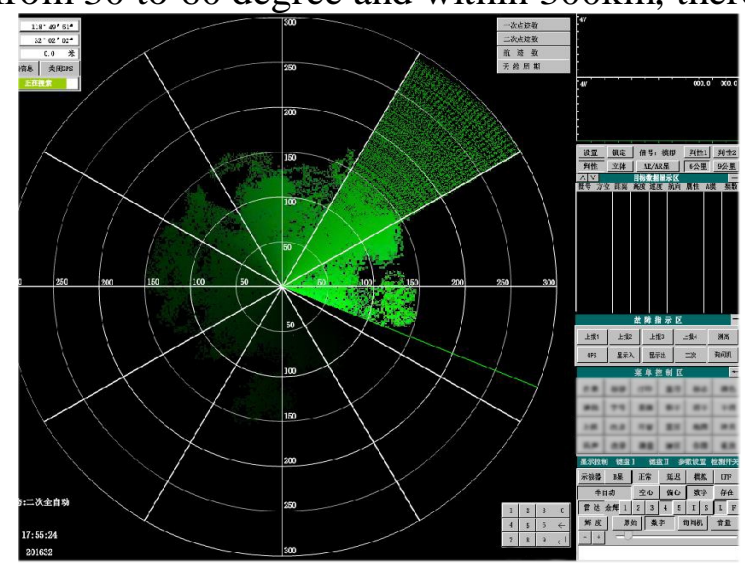

Fig.6. Barrage Jamming on the Terminal Displayer

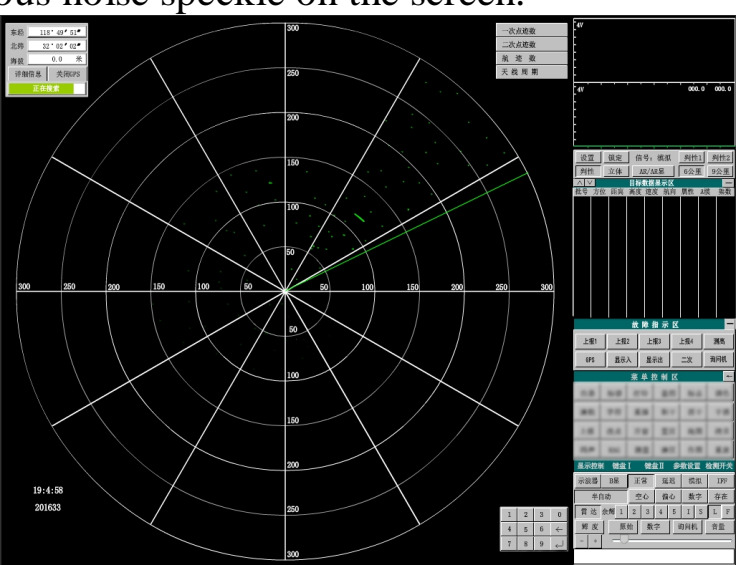

Fig.7. Output of RITL model with Correct Solution

According to the given barrage jamming scenario, through the training simulation process with RITL model in the correct solution on all components, the output image is shown as Fig.7. Where near the radar site, the ground clutter is suppressed efficiently. In addition, from 30 to 60 azimuth 
degree and within $300 \mathrm{~km}$, the noise speckle energy has been largely cancelled. The residual speckle energy depends on the operation results by different radar terminal trainees, in other words, depends on different solutions of RITL model.

\section{Conclusion}

RITL model is essential for a man-in-the-loop radar training simulated system in contrast to typical models such as PDW, barrage jamming and power plus frequency ECCM models for campain simulation since the later serves computer-generated-force pattern. The experimental results for a $\mathrm{L}$ band surveillance radar indicate the effectiveness of interaction architecture for a radar operational training system with RITL model. Future work would probably focus on optimizing components or parameters in RITL model and radar ECCM models.

\section{References}

[1] Xiaojun Wang, Xiaoyong Lu, etc., Models Design for Surveillance Radar Electronic Counter-Countermeasure Interaction Training System, International Conference on Electrical Engineering and Mechanical Automation, ICEEMA 2015, June 13-14, 2015, Suzhou, China.

[2] Lu Xiaoyong, Yan Shusheng, etc. Modeling of Analog Radar Pulse-width in EM Environment and Its Simulation [J]. Journal of Air Force Early Warning Academy, 2013, 27(2): 112-114.

[3] Xiaojun Wang, Quansheng Li, Xiaoyong Lu. Simulation for Land based Surveillance Radar Electromagnetic Environment by Typical Pulse Word Characteristics. International Conference on Control Engineering and Communication Technology, 7-9 December, 2013, Xiangtan, China.

[4] Xiaojun Wang, Xiaoyong Lu , Shusheng Yan ,et. al, Modeling and Simulation for Electromagnetic Interaction Training System of Surveillance Radar in Jamming Environment, 2014 International Conference on Computer Science and Technology, August 16-17, 2014, Kunming, China.

[5] Stephen L. Johnston. Radar electronic counter-countermeasures[J]. IEEE Transactions on Aerospace and Electronic Systems, 1987, 14 (1):109-117.

[6] Yin Kang-Yin, WANG Qian, WANG Guang, Research on the Military Conception Model of Air Defense Early Warning System[J]. Journal of Air Force Early Warning Academy, 2014, 27(2): 112-114

[7] Hongyan Deng, Guilong Deng . Warfighting Simulation Theories and Practices[M].National Defence Industry Press.Beijing,2013:20-22.

[8] Lenghui He, etc. Fundamentals of Operational Simulation[M]. National Defence Industry Press.Beijing,2013:20-22. 